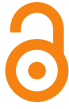

Recibido: 6 agosto 2021

Revisión: 18 noviembre 2021

Aceptado: 27 diciembre 2021

\section{Dirección autoras:}

${ }^{1}$ Universidad Insurgentes (UIN) Plantel Sur I, Calz. de Tlalpan 1064, Nativitas, Benito Juárez, 03500 Ciudad de México (México)

${ }^{2}$ Facultad de Estudios Superiores Zaragoza, Universidad Nacional Autónoma de México. Av. Guelatao 66, Ejército de Oriente Indeco II Issste, Iztapalapa, 09230 Ciudad de México (México)

\section{E-mail / ORCID}

\section{psico.paolatirado@gmail.com}

https://orcid.org/0000-00019620-1031

\section{roquehp@yahoo.com.mx}

(iD) https://orcid.org/0000-00033902-6585
ARTÍCULO / ARTICLE

\section{Validación de la Escala Uso y Función de las TIC en Contextos Educativos para estudiantes de Educación Superior}

\author{
Validation of the Use and Function of ICT in \\ Educational Contexts Measurement Scale for \\ students in Higher Education
}

Paola Jazmín Tirado Lara ${ }^{1}$ y María del Pilar Roque Hernández ${ }^{2}$

Resumen: La importancia de las TIC en la Educación Superior está presente en las políticas internacionales y nacionales; en momentos contextuales donde se prioriza la educación a distancia y asíncrona, es urgente identificar las competencias de los universitarios. Esta investigación buscó validar en población mexicana una escala para evaluar la frecuencia de uso y función de las TIC y los contextos educativos en donde se utilizan, que apoye el conocimiento acerca de sus fortalezas y áreas de oportunidad. La metodología fue cuantitativa y se dividió en dos fases: 1) estudio descriptivo para diseñar y pilotear la escala; y 2) validación de la versión final del instrumento con 390 estudiantes de tres estados del país. A partir del análisis de estudios descriptivos, de normalidad, validez de constructo (análisis factorial) y confiabilidad, el instrumento quedó conformado por dos componentes: a) 26 reactivos distribuidos en 4 factores que miden frecuencia de uso y función; y b) 8 reactivos de opción múltiple para identificar los contextos educativos de uso. La escala explica $42.7 \%$ de la varianza, con un Alpha de Cronbach global de .91. Emplear instrumentos confiables de evaluación en la Educación Superior, facilitará la intervención y el desarrollo de estrategias psicoeducativas para la adquisición de competencias en TIC.

Palabras clave: Tecnología de la Información, Competencia Digital, Evaluación Educativa, Educación Superior, Usos de la Tecnología en la Educación.

Abstract: The importance of ICT in Higher Education is present in international and national policies; in contextual moments where distance and asynchronous education is prioritized, it is urgent to identify the competences in university students. This research sought to validate in the Mexican population a scale to evaluate the frequency of use and function of ICTs and the educational contexts in which they are used, that supports knowledge about their strengths and areas of opportunity. The methodology was quantitative and divided into two phases: 1) descriptive study to design and pilot the scale; and 2) validation of the final version of the instrument with 390 students from three states of the country. From the analysis of descriptive studies, normality, construct validity (factor analysis) and reliability, the instrument was make up of two components: a) 26 items distributed in 4 factors that measure frequency of use and function; and b) 8 multiple - choice items to identify educational contexts of use. This scale explained $42.7 \%$ of the variance, with a global Cronbach Alpha of .91. The use of reliable assessment tools in higher education, will facilitate the intervention and the development of psycho - educational strategies for the acquisition of ICT skills.

Keywords: Information Technology, Digital Competence, Educational Assessment, Higher Education, Technology Uses in Education. 


\section{Introducción}

Las Tecnologías de la Información y la Comunicación (TIC) son herramientas que permiten la producción, almacenamiento y manejo de la información, posibilitando así la interacción, el trabajo colaborativo, la comunicación y difusión de la información (Dele-Ajayi et al., 2021; Ramos et al., 2019); para Olaya y Peirano (2007) las TIC surgieron de la convergencia entre las tecnologías desarrolladas en las áreas de las telecomunicaciones, la computación y la microelectrónica.

Entre las características de las TIC (Cabero y Llorente, 2015) se encuentran: a) inmaterialidad, al generar y procesar información digital; b) interactividad entre la persona y el equipo informático; c) instantaneidad, pues se pueden emplear sin importar lugar y tiempo; d) innovación, cuando se mejora y modifica cada generación tecnológica; e) digitalización de sus elementos visuales y auditivos; $f$ ) interconectividad entre TIC; y g) diversidad, respecto a las funciones a desempeñar. Las TIC, también han sido tipificadas, a partir del tipo de herramientas que representan; Mengual et al., (2013) las clasificaron en: 1) redes de telecomunicaciones, como las redes telefónicas o la banda ancha; 2) terminales, que refieren al hardware y software que fungen de soporte a los servicios de las TIC (e.g. computadora, teléfono inteligente, sistema operativo); y 3) servicios, que incluyen plataformas, comunidades virtuales e hipermedias que ofrecen las TIC, entre ellas, correo electrónico, wikis, plataformas educativas, blogs y redes sociales.

Se han documentado diversas ventajas del empleo de las TIC en la educación, entre ellas, que ofrecen novedosas posibilidades para aprender a aprender y para dirigir el propio aprendizaje (Dávila y Gutiérrez, 2019; Dele-Ajayi et al., 2021; Recio et al., 2020). Para la Organización de las Naciones Unidas para la Educación la Ciencia y la Cultura (UNESCO, 2019) las TIC pueden complementar, enriquecer y transformar la educación, por ello, la misma UNESCO, estimó que para 2030 las tecnologías se utilizarán para fortalecer las competencias docentes, así como para documentar y difundir entre docentes y alumnos las prácticas idóneas para utilizarlas, con especial atención a las innovaciones en el ámbito digital.

Sin embargo, todavía hay mucho por hacer para que las TIC adquieran un mayor rol e importancia en la educación; al respecto, Prendes et al., (2018) identificaron en 2,054 estudiantes de 79 universidades de España, que no habían integrado el uso de las TIC en ámbitos académicos, pese a que las utilizaban para comunicarse en lo personal y para actividades de ocio, desmitificando así, el considerarlos nativos digitales. No obstante, Dávila y Gutiérrez (2019) justificaron que los alumnos son nativos digitales, pues mantienen un vínculo estrecho con las TIC, mismas que contribuyen a ampliar su vida social y también porque las emplean como herramientas indispensables en los escenarios de aprendizaje.

En México, Madrid et al., (2016) realizaron un metaanálisis sobre la educación mediada por las TIC, a partir de la revisión en bases de datos y sitios web especializados, considerando artículos de revistas, ponencias de congresos y tesis de 2004 a 2014, entre los aspectos identificados están: a) 49\% de las investigaciones, se han realizado en el nivel superior; y b) no constituye una línea prioritaria de generación y aplicación del conocimiento, cuestiones como el desarrollo, implementación y evaluación de materiales y recursos educativos digitales, así como la caracterización de 
los factores psicoeducativos y sociales de los sistemas y ambientes educativos. Por su parte, Islas et al., (2016) realizaron una investigación cuantitativa y descriptiva para identificar los patrones de uso de las TIC en 879 universitarios de instituciones privadas y públicas: $66 \%$ de los universitarios ( $11 \%$ hombres) podían aprender con o sin el uso de las TIC; aunque el 34\% restante (hombres), eran dependientes de los medios tecnológicos. En específico, 34\% de las mujeres utilizaba herramientas tecnológicas, algunas veces para realizar organizadores gráficos o textuales, o para elaborar actividades de aprendizaje colaborativo y casi siempre para perfeccionar sus conocimientos y favorecer el aprendizaje sobre un tema.

Otra investigación reportada fue la de Olivares et al., (2016) quienes identificaron que los estudiantes de Educación Superior en el país, pese a la competencia digital que poseen, carecen de habilidades para aplicarlas a aspectos de investigación, gestión, procesamiento y análisis de información; además, de emplear pocos recursos como editores Web, video conferencias y listas de distribución, por lo que necesitan actualizar sus conocimientos sobre las TIC y desarrollar mayor competencia digital.

Los hallazgos sobre el tema, coinciden en la contradicción de la pericia en el uso de las TIC por parte de Universitarios en su vida personal en contraste a la esfera académica, ello puede en las estadísticas oficiales; de acuerdo con el Instituto Nacional de Estadística y Geografía (INEGI, 2020) existe un mayor uso del internet conforme aumenta el grado de estudios: $59.1 \%$ de usuarios con educación básica, $91.8 \%$ del total de personas que había cursado hasta la educación media superior y $96.4 \%$ de usuarios con Educación Superior; sin embargo el uso que le dan a las misma fueron: entretenimiento (91.5\%); obtención de información $(90.7 \%)$; comunicación con otras personas (90.6\%); y acceso a redes sociales (87.8\%).

Ante este panorama, se observa un área de oportunidad para favorecer que los estudiantes, usen las TIC como una herramienta para indagar mediante métodos científicos, explicar el mundo, diseñar y producir prototipos tecnológicos y ser críticos sobre cuestiones científicas y tecnológicas (Dávila y Gutiérrez, 2019). Las TIC pueden favorecer el desarrollo de diversas competencias en los universitarios, es decir la relación entre conocimientos - habilidades - actitudes (Gordon et al., 2010; Recio et al., 2020) y de manera más específica, las TIC son vitales en el desarrollo de la competencia digital.

Para la Unión Europea (2018) la competencia digital es clave para los ciudadanos y en específico para los profesionales del siglo XXI, pues facilitarán participar en la sociedad y el aprendizaje durante la vida. Para la Comisión Europea (2019) la competencia digital abarca los conocimientos, habilidades y actitudes que involucran: 1) búsqueda, evaluación y retroalimentación de la información; 2) colaboración entre tecnologías y canales digitales; 3 ) netiqueta, que tiene que ver con la participación ciudadana e identidad digital; 4) protección y seguridad de datos, servicios y medio ambiente; 5) resolución de problemas; y 6) creación de contenidos. La misma Comisión Europea subrayó que la competencia digital que desarrollan los universitarios, debe estar orientada a los ámbitos laboral, académico y funcional en la vida cotidiana.

Por tanto, la relación entre TIC y educación debe ser analizada en los diferentes contextos educativos; para Trilla et al., (2003) hay que referirse a tres contextos de 
educación: a) formal, que apunta al sistema educativo institucionalizado que otorga grados o niveles educativos (e.g. escuela); b) los no formales, los cuales tienen que ver con actividades organizadas y sistemáticas, que se realizan fuera del marco del sistema oficial de educación (e.g. casas de cultura), por lo cual no dan salida a niveles educativos; y c) los informales, vinculados al proceso educativo inmerso en realidades culturales como el hogar. En particular, el uso de las TIC por parte de los universitarios en contextos formales y no formales de la educación, puede favorecer en los estudiantes; 1) el desarrollo de la alfabetización digital acorde con las demandas globales en el campo de trabajo (UNESCO, 2014); 2) la resolución de problemas, creación de contenidos, comunicación en línea, cuidado y seguridad digital (European Commission, 2019); y 3) el desarrollo de competencias relacionados con contenidos específicos de los planes y programas de estudios en la Educación Superior (Ramos et al., 2019).

Resulta claro que, con la inclusión de las TIC tanto en la enseñanza como en el aprendizaje, surgen nuevos requerimientos en la formación de los estudiantes, por lo que sigue vigente la necesidad de promover en ellos la competencia digital, orientada no sólo al ámbito formal de educación, sino a todos los ámbitos en que interactúan y se desenvuelven. De ahí que para promover en México la competencia digital de los universitarios, se precisa, evaluar su nivel de dominio, con qué competencias digitales cuentan, e identificar los usos, la función y los ámbitos en los cuales las ponen en práctica; al respecto Olivares et al., (2016) indicaron que valorar la competencia digital de los universitarios, facilitará identificar las áreas de oportunidad para su formación, proponer estrategias para adquirirlas o fortalecerlas y permitirá aprovechar de mejor forma los recursos de las instituciones educativas.

Entre los métodos para evaluar la competencia digital, están las entrevistas, la observación y los instrumentos electrónicos o no, ya sean formales o informales, como las escalas de percepción. Uno de ellos es el TICómetro (Coordinación de Tecnologías para la Educación, 2020) que se ocupa desde 2012 con estudiantes de la UNAM, para identificar el nivel de habilidades en relación con la tecnología y para formular una estrategia de integración de TIC en las actividades educativas. Se evalúan temas relacionados con la: búsqueda, selección y validación de la información; procesamiento y administración de la información; comunicación y colaboración en línea; y seguridad; incluye 30 reactivos (opción múltiple, preguntas de respuesta construida; y simuladores para la resolución de actividades concretas) y 10 ítems para conocer sobre acceso y hábitos de uso de las TIC.

Otro instrumento en proceso es el Perfil de Competencia Digital (Olivares et al., 2016) validado con 585 estudiantes de una universidad en Sonora (México). A través del análisis factorial exploratorio, la escala quedó conformada por 45 reactivos: 12 de datos generales y 33 sobre competencia digital distribuidos en las subescalas dominio tecnológico $(a=.90)$, administración de recursos digitales $(a=.87)$ y motivación hacia el uso de las TIC $(a=.84)$.

Otro instrumento es el de Jiménez y Alvarado (2017) quienes validaron un cuestionario para medir la frecuencia y ámbito de uso de las TIC en 178 estudiantes españoles, la escala final contó con 28 reactivos distribuidos en tres dimensiones, la primera media el uso de las TIC en ámbitos relacionados con juegos y mensajería en el celular y un computador, la segunda media el uso de las TIC en teléfonos o 
computadores en el ámbito educativo y la tercera media la emoción relacionada con el uso de las TIC; la escala obtuvo un alpha de cronbach total $=.86$.

A partir de la revisión presentada, es clara la importancia de las TIC en la Educación Superior, así como la necesidad de identificar y desarrollar competencias digitales en los profesionales en formación. Al respecto, Recio et al. (2020) realizaron un meta-análisis para cuantificar de 2014 a 2019 las investigaciones publicadas en la Web of Science, sobre evaluación de competencias digitales en universitarios; sus hallazgos mostraron la presencia de 18 artículos en su mayoría cuantitativos, dirigidos a identificar a través de instrumentos formales o informales (cuestionarios, test o pruebas de rendimiento), una dimensión perceptiva (entre ellos, actitudes, utilidad y uso de las TIC) o bien, una de rendimiento o cognitiva (habilidades, alfabetización digital, logro) y sólo 5 de dichos trabajos, evaluaban ambas dimensiones. Recio et al. también indicaron que pese a que, los instrumentos identificaban conocimientos, habilidades y actitudes acerca de las competencias digitales, no priorizaban realizar su validación y carecían de sistematización adecuada, obstaculizando entender qué aspectos deben considerarse en el concepto de competencia digital.

Actualmente en México, debido a la suspensión de actividades académicas presenciales vinculada con la emergencia sanitaria global por COVID-19, el Gobierno Federal a través de la Comisión Nacional para la Mejora Continua de la Educación (2020) ha sugerido asuntos como diversificar las alternativas de educación en línea según los dispositivos disponibles en diferentes contextos y sus condiciones de uso, así como fortalecer la educación a distancia y focalizada. Para ello, ha sido fundamental que las instituciones de Educación Superior implementen medidas para adaptarse a las nuevas circunstancias (Asociación Nacional de Universidades e Instituciones de Educación Superior, 2020) de tal modo que se ha transitado de la modalidad escolarizada y presencial a la no presencial.

De acuerdo con la UNESCO (2020) casi $80 \%$ de la población estudiantil en el mundo ha sido afectada por el cierre de las escuelas, lo que se traduce en 1,370 millones de alumnos; ante esta situación, las TIC han facilitado continuar con las actividades docentes y la comunicación sincrónica y/o asincrónica de los actores involucrados. No obstante, se precisa seguir realizando acciones que faciliten la enseñanza, la implicación activa en el aprendizaje y el acceso a la información y adquisición de competencias en beneficio de la formación personal y profesional del individuo, entre ellas, innovar en el uso de las TIC, emplear mayormente la creatividad y desarrollar la responsabilidad y autodeterminación de los involucrados en el proceso educativo.

Para Dávila y Gutiérrez (2019) incorporar a las TIC en el aprendizaje a distancia, influye en que éste sea significativo y en la capacidad analítica de los alumnos, en cuestiones como elegir los recursos tecnológicos más adecuados para satisfacer sus necesidades de aprendizaje, aprender a utilizarlos de forma responsable y obtener la información más adecuada. En este sentido, estas tecnologías representan herramientas de aprendizaje y el alumno para beneficiarse de ellas, debe contar con cierta competencia digital y asumir un rol activo en su aprendizaje, por lo que contar con instrumentos formales, confiables y válidos es vital para la adecuación de la educación mexicana a las nuevas exigencias en la educación. 
Ahora bien, para promover en México la competencia digital de los universitarios, se precisa, evaluar su nivel de dominio, con qué competencias digitales cuentan, e identificar los usos, la función, frecuencia y los ámbitos en los cuales las ponen en práctica, porque si bien, los instrumentos antes descritos evalúan la competencia digital, no la contextualizan en los diferentes escenarios educativos (Trilla, et al., 2003), ello pone de manifiesto la necesidad de contar con instrumentos confiables que entienda los usos de las TIC más allá del sistema institucionalizado. Cabe resaltar, que pese a la mayor accesibilidad que hay hacia las TIC (INEGI, 2020) su uso todavía dista de vincularse con el ámbito educativo y sigue altamente relacionado con el entretenimiento y comunicación (INEGI, 2020) por lo que una ventaja de conocer los hábitos de uso de las TIC de los estudiantes fuera del contexto educativo formal, permitirá reenfocar las habilidades digitales de los estudiantes de un contexto informal a uno institucionalizado, lo que a su vez, facilitará identificar áreas de oportunidad y desarrollar de estrategias que permitan a las Instituciones Educativas mejorar y optimizar sus propios recursos.

En cuanto a otros instrumentos relacionados con las TIC en México, existen diversos métodos de evaluación que si bien aportan gran información descriptiva sobre la competencia digital, no son instrumentos formales que cuenten con un proceso establecido para la validación (Coordinación de Tecnologías para la Educación, 2020) o restringen el análisis de la competencia digital a un único contexto educativo, sin contemplar el acceso y hábitos de uso de las TIC (Olivares et al., 2016) por lo que se requiere de instrumentos formales que puedan complementar una evaluación objetiva sobre el uso de las TIC en los estudiantes.

A partir do anterior, el presente trabajo tuvo por objetivo validar una escala para evaluar la frecuencia de uso y función de las TIC, así como los contextos educativos en donde los universitarios las utilizan, que apoye el conocimiento acerca de sus competencias digitales, áreas de oportunidad y que dirija la intervención educativa. El estudio fue exploratorio descriptivo, realizado con metodología cuantitativa e incluyó dos fases: en la primera, a través de un diseño de investigación longitudinal, se construyó el instrumento y en la segunda fase, a partir de un diseño transversal se obtuvo su validez de constructo, confiabilidad y percentiles. Esta investigación se realizó como parte del Proyecto de Investigación UNAM/FESZ/PSIC/279/2020.

\section{Método}

\subsection{Fase 1. Construcción del instrumento}

Se trabajó en una universidad pública ubicada en una zona urbana con un nivel medio - alto de marginación (Sistema de Información del Desarrollo Social, 2018) en la Ciudad de México. Para el ciclo escolar 2020, dicha institución tenía una matrícula total de 12,359 estudiantes distribuidos en nueve planes de estudio a nivel pregrado; la licenciatura en Psicología contaba con una matrícula de 2,227 estudiantes, de los cuales 1,673 estaban inscritos en alguna de las 4 áreas de profesionalización (educativa, clínica, social y laboral) (Hernández, 2020). Participaron de manera voluntaria 17 estudiantes (edad $M=21.29, \mathrm{DE}=1.40$, Rango $=20-26$ ) pertenecientes al octavo semestre de la Carrera de Psicología, área educativa (5 hombres y 12 mujeres) cuyo promedio escolar se ubicó en 8.235 (DE = 1.001). Y, también, una juez, docente 
investigadora experta en psicometría y educación (55 años y 31 de antigüedad docente). El muestreo fue no probabilístico intencional.

Los instrumentos empleados fueron: versiones preliminares de la Escala Uso y Función de las TIC en Contextos Educativos, hojas de registro, bitácoras y datos estadísticos oficiales de la institución escolar. Se diseñó la primera versión del instrumento a partir de la revisión bibliográfica y la lluvia de ideas de los investigadores; los reactivos incluidos, se agruparon en dos componentes. Los principales preceptos éticos considerados en el piloteo del instrumento, fueron el consentimiento informado, la confidencialidad y el anonimato. Se realizaron adecuaciones a esta primera versión, principalmente en cuanto al lenguaje, datos de identificación, establecimiento de componentes, así como la inclusión y reubicación de reactivos.

\subsection{Fase 2. Validación del instrumento}

Se trabajó en tres universidades, 1 pública y 2 privadas. La universidad pública fue descrita en la Fase 1. La segunda, correspondió a una institución privada ubicada en una zona urbana con bajo nivel de marginación en el municipio de Chalco, Estado de México (Secretaría de Desarrollo Social, 2013). La matrícula era de 609 alumnos distribuidos en 5 licenciaturas; para el año 2019, la carrera de Psicología contaba con 138 alumnos (Secretaría de Economía, Datawheel, 2020). La tercera universidad, estaba ubicada en una comunidad semi rural de alta marginación enclavada en las montañas de Chiapas (Consejo Nacional de Población, 2015); contaba con una población total de 850 alumnos inscritos en 4 carreras a nivel de posgrado y 13 licenciaturas de las áreas social, clínica, laboral y educativa; en esta última, estaban inscritos 200 estudiantes.

Participaron de forma voluntaria 390 universitarios (edad $\mathrm{M}=21.44$ años, $\mathrm{DE}=$ 2.008, Rango $=17$ - 32) de los cuales: $71.3 \%$ eran mujeres; $89 \%$ no debía materias; y en general, el promedio de calificaciones del semestre anterior cursado fue 8.99 (DE = .56). Del total, $79.2 \%$ pertenecían a la escuela pública, $12.8 \%$ a la universidad en el Estado de México y $7.9 \%$ a la universidad de Chiapas. Para la selección de los participantes se utilizó un muestreo no probabilístico e intencional. Se utilizó la escala construida en la Fase 1; además de bitácoras y datos estadísticos oficiales de las instituciones educativas. La escala se aplicó primordialmente de forma grupal dentro de las instituciones y a partir del consentimiento informado de los participantes. Al finalizar la aplicación, se verificó que todos los reactivos y datos socioeconómicos tuvieran respuesta; el tiempo de aplicación promedio, fue 10 minutos. Para el análisis de los datos, se diseñó una base en el programa SPSS Ver. 24 en la cual se realizaron diferentes procedimientos: obtención de datos descriptivos, estudios de normalidad (Kolmogorov Smirnov), error de medida, validez de constructo (análisis factorial exploratorio), consistencia interna (Alpha de Cronbach) y obtención de percentiles.

\section{Resultados}

\subsection{Fase 1. Construcción del instrumento}

La versión final del instrumento, quedó conformado por tres secciones: 1) datos sociodemográficos; 2) instrucciones para su respuesta; y 3) 46 reactivos para evaluar dos componentes: (a) Frecuencia de Uso y Función de las TIC. Refiere a la frecuencia y 
utilidad que los estudiantes dan a las TIC en una semana. Incluye 38 reactivos que se evalúan de forma cuantitativa distribuidos en 4 dimensiones (Tabla 1) y presentados en escala tipo Likert de 4 puntos ( $1=0$ a 1 vez por semana - $4=$ más de 5 veces a la semana). (b) Contextos Educativos de Uso de las TIC. Incluye 8 reactivos, cada uno con 4 opciones de respuesta, para identificar cualitativamente los contextos en los cuales los universitarios cotidianamente prefieren emplear las $\mathrm{TIC}$, a partir de aspectos como comodidad, acceso, preferencia o disponibilidad: 1) formal (universidad/escuela); 2) no formal (centros educativos ajenos a la universidad en la cual se está inscrito: casa de cultura o museos); y (c) informal (casa y espacio público como cibercafé).

Tabla 1. Dimensiones del componente Frecuencia de uso y función de las TIC

\begin{tabular}{|c|c|c|}
\hline Dimensión & Definición operacional & Reactivos \\
\hline \multirow[t]{9}{*}{$\begin{array}{l}\text { Académico- } \\
\text { individual }\end{array}$} & \multirow{9}{*}{$\begin{array}{l}\text { Hábitos y usos de las TIC } \\
\text { en actividades } \\
\text { individuales vinculadas } \\
\text { con el contexto formal } \\
\text { de educación }\end{array}$} & $\begin{array}{l}\text { 2. Desarrollar actividades académicas } \\
\text { individualmente }\end{array}$ \\
\hline & & $\begin{array}{l}\text { 4. Encontrar información en internet } \\
\text { relacionada con mi vida académica }\end{array}$ \\
\hline & & 8. Buscar información escolar \\
\hline & & $\begin{array}{l}\text { 12. Usar aplicaciones que me ayuden en mis } \\
\text { prácticas profesionales }\end{array}$ \\
\hline & & 14. Recibir clases en línea \\
\hline & & $\begin{array}{l}\text { 17. Ver videos en línea que hablen sobre } \\
\text { temáticas que se abordan en mis clases }\end{array}$ \\
\hline & & $\begin{array}{l}\text { 25. Diseñar y preparar mis exposiciones de } \\
\text { clase }\end{array}$ \\
\hline & & $\begin{array}{l}\text { 31. Usar aplicaciones que me ayuden a } \\
\text { desarrollar los conocimientos que requiero } \\
\text { para mi desarrollo académico }\end{array}$ \\
\hline & & 38. Realizar exámenes en línea \\
\hline \multirow[t]{9}{*}{$\begin{array}{l}\text { Académico- } \\
\text { compañeros }\end{array}$} & \multirow{9}{*}{$\begin{array}{l}\text { Hábitos y usos de las TIC } \\
\text { en actividades entre } \\
\text { pares en actividades } \\
\text { relacionadas con el } \\
\text { contexto formal de } \\
\text { educación }\end{array}$} & $\begin{array}{l}\text { 1. Desarrollar actividades académicas en } \\
\text { equipo }\end{array}$ \\
\hline & & $\begin{array}{l}\text { 7. Preguntar a mis compañeros dudas } \\
\text { referentes a las tareas escolares }\end{array}$ \\
\hline & & $\begin{array}{l}\text { 9. Participar en videoconferencias (ejemplo en } \\
\text { congresos o clases) }\end{array}$ \\
\hline & & $\begin{array}{l}\text { 15. Ponerme de acuerdo con mis compañeros } \\
\text { de clase para realizar un trabajo escolar }\end{array}$ \\
\hline & & $\begin{array}{l}\text { 20. Realizar en línea documentos de alguna } \\
\text { asignatura }\end{array}$ \\
\hline & & 24. Dar y recibir avisos referentes a las clases \\
\hline & & $\begin{array}{l}\text { 28. Recibir información del maestro sobre las } \\
\text { actividades de la clase }\end{array}$ \\
\hline & & $\begin{array}{l}\text { 32. Compartir o apreciar materiales multimedia } \\
\text { durante las clases (películas, música, imágenes, } \\
\text { etc) }\end{array}$ \\
\hline & & $\begin{array}{l}\text { 37. Compartir material complementario } \\
\text { referente a alguna asignatura }\end{array}$ \\
\hline
\end{tabular}




\begin{tabular}{|c|c|c|}
\hline Dimensión & Definición operacional & Reactivos \\
\hline \multirow[t]{10}{*}{$\begin{array}{l}\text { No académico- } \\
\text { individual }\end{array}$} & \multirow{10}{*}{$\begin{array}{l}\text { Hábitos y usos de las TIC } \\
\text { en actividades } \\
\text { individuales relativas a } \\
\text { la educación informal }\end{array}$} & $\begin{array}{l}\text { 3. Tomar decisiones personales respecto a mi } \\
\text { vida cotidiana }\end{array}$ \\
\hline & & $\begin{array}{l}\text { 5. Revisar información que no esté relacionada } \\
\text { con mi formación profesional }\end{array}$ \\
\hline & & $\begin{array}{l}\text { 11. Buscar tutoriales (ejemplo YouTube) para } \\
\text { realizar actividades ajenas a la escuela } \\
\text { (ejemplo. reparar mi celular) }\end{array}$ \\
\hline & & 16. Comprar en línea \\
\hline & & $\begin{array}{l}\text { 19. Ver series, videos o películas a través de } \\
\text { algún dispositivo electrónico }\end{array}$ \\
\hline & & 22. Descargar música o libros \\
\hline & & $\begin{array}{l}27 . \text { Revisar las redes sociales como medio } \\
\text { recreativo }\end{array}$ \\
\hline & & 30. Escuchar música \\
\hline & & 33. Tomarme fotos \\
\hline & & $\begin{array}{l}\text { 35. Hacer mis propios videos, publicidad, } \\
\text { documentos, etc. }\end{array}$ \\
\hline \multirow{10}{*}{$\begin{array}{l}\text { No académico- } \\
\text { compañeros }\end{array}$} & \multirow{10}{*}{$\begin{array}{l}\text { Hábitos y usos de las TIC } \\
\text { entre pares en } \\
\text { actividades de la } \\
\text { educación informal (no } \\
\text { escolar) }\end{array}$} & 6. Chatear con mis amigos \\
\hline & & $\begin{array}{l}\text { 10. Participar en grupos de debate no } \\
\text { relacionados a temáticas escolares }\end{array}$ \\
\hline & & $\begin{array}{l}\text { 13. Invitar a mis amigos a algún evento (fiestas, } \\
\text { conciertos) }\end{array}$ \\
\hline & & $\begin{array}{l}\text { 18. Compartir música, fotos libros y/o videos } \\
\text { fuera de clase }\end{array}$ \\
\hline & & $\begin{array}{l}\text { 21. Etiquetar a mis amigos en comentarios que } \\
\text { les pueden interesar o ser graciosos }\end{array}$ \\
\hline & & $\begin{array}{l}\text { 23. Etiquetar a mis amigos en vídeos que no } \\
\text { han visto }\end{array}$ \\
\hline & & 26. Usar aplicaciones o páginas de citas \\
\hline & & $\begin{array}{l}\text { 29. Contestar y/o reaccionar a comentarios y } \\
\text { vídeos de mis amigos }\end{array}$ \\
\hline & & 34. Jugar en línea contra otras personas \\
\hline & & $\begin{array}{l}\text { 36. Organizar o compartir eventos sociales } \\
\text { (fiestas) }\end{array}$ \\
\hline
\end{tabular}

\subsection{Fase 2. Validación del instrumento}

Con la versión ya validada del instrumento y para el componente Frecuencia de Uso y Función de las TIC, se obtuvieron las puntuaciones totales de la muestra ( $\mathrm{DE}=7.579$, Rango $=45-81$ ) identificándose que en promedio, los participantes utilizaban las TIC de 4 a 5 veces por semana. En cuanto a las dimensiones, se encontró: 1) No Académico-Compañeros (Total $M=21.94$ puntos, $D E=3.61$ ); 2) AcadémicoCompañeros (total $M=18.26$ puntos, $D E=4.46) ; 3$ ) No académico-Individual (Total $M=$ 13.26 puntos, $D E=2.16$ ); y 4) Académico-Individual ( 
anterior refleja que los participantes utilizaban las TIC de 2 a 3 veces por semana tanto para actividades académicas individuales o en grupo como para aquellas realizadas fuera del ámbito académico. En la Tabla 2 se incluye la media y desviación estándar de cada uno de los reactivos finales para este componente.

Tabla 2. Media y desviación estándar de las puntuaciones totales por reactivo.

\begin{tabular}{cccccc}
\hline Reactivo & Media & DE & Reactivo & Media & DE \\
\hline 1 & 2.35 & .93 & 19 & 2.88 & 1.048 \\
2 & 3.17 & .881 & 20 & 2.76 & .978 \\
4 & 2.95 & .975 & 21 & 2.71 & 1.155 \\
5 & 3.41 & .904 & 22 & 2.59 & 1.044 \\
6 & 2.78 & .927 & 23 & 2.17 & 1.111 \\
7 & 2.78 & .927 & 24 & 2.44 & .973 \\
8 & 3.24 & .809 & 25 & 2.67 & .890 \\
11 & 2.37 & 1.042 & 27 & 3.04 & .981 \\
12 & 2.21 & .935 & 28 & 2.50 & .909 \\
13 & 1.88 & 1.011 & 29 & 2.71 & 1.074 \\
15 & 2.75 & .874 & 30 & 3.22 & 1.012 \\
17 & 2.03 & .925 & 31 & 2.43 & .967 \\
18 & 2.70 & 1.058 & 32 & 2.27 & 1.057 \\
\hline
\end{tabular}

Nota. 1 = nunca a una vez por semana, $2=2$ a 3 veces por semana, $3=4$ a 5 veces por semana, $4=$ más de 5 veces a la semana. Elaboración propia.

En cuanto al componente Contextos Educativos de Uso de las TIC, 92.6\% de la muestra prefería utilizarlas en un contexto no formal, $1.9 \%$ las utilizaba en el informal o formal respectivamente y $.6 \%$ en el espacio público. También se identificó la preferencia por usarlas en más de un contexto: $1.9 \%$ prefería hacerlo en la escuela y su hogar; . $3 \%$ las utilizaba en la escuela y en una casa de cultura; $.3 \%$ en una casa de cultura y hogar y .3\% en el hogar y espacio público.

La Escala Uso y Función de las TIC en Contextos Educativos, evalúa dos componentes (uno cuantitativo y otro cualitativo) a través de 34 reactivos. Tras realizar el análisis de normalidad a las puntuaciones obtenidas, se obtuvo un valor $p=.001$, lo que refiere a una muestra no paramétrica. En relación con la dispersión de los datos debido al error de medición, se identificó un error bajo $(E S=.95)$. Previo al análisis factorial, se analizó la matriz con la prueba de esfericidad de Bartlett en la que se obtuvo un valor $p=.00$ que indicó una adecuada distribución de la varianza; con la medida Kaiser-Meyer-Olkin de adecuación de muestreo, se obtuvo un valor KMO $=.911$, correspondiente a una relación alta entre las variables.

Para la validez de constructo, se llevó a cabo un análisis factorial exploratorio con los 38 reactivos iniciales; el método de extracción fue por máxima verosimilitud y la rotación VARIMAX. Se excluyeron los reactivos que obtuvieron un índice de correlación de componentes menor a .45; tras este análisis, se eliminaron 12 reactivos. Se obtuvo una estructura final compuesta por cuatro factores y 26 reactivos (positivos y negativos); dichos factores explican el $42.7 \%$ de la varianza. El factor que más varianza explica, fue el No académico - compañeros (Tabla 3). 
Tabla 3. Caracterización de los factores de Frecuencia de Uso y Función de las TIC

\begin{tabular}{lccc}
\hline \multicolumn{1}{c}{ Factor } & $\begin{array}{c}\text { Reactivos } \\
\text { positivos }\end{array}$ & $\begin{array}{c}\text { Reactivos } \\
\text { negativos }\end{array}$ & $\begin{array}{c}\text { Porcentaje de } \\
\text { varianza explicada }\end{array}$ \\
\hline No académico-compañeros & $13,18,19,21,23$, & $22,30,32$ & 26.70 \\
Académico-compañeros & $1,7,15,20,24$, & --- & 6.908 \\
No académico-individual & 25,28 & & 4.931 \\
Académico-individual & 5,27 & $2,4,6,8$ & 4.161 \\
\hline
\end{tabular}

En la Tabla 4 se muestran los valores de las comunalidades entre cada reactivo y el factor al que pertenecen, al analizar todas las comunalidades se obtuvo una comunalidades $M=.517$ y un Rango = .35 - .78 $(D E=.110)$ indicando que los reactivos son una adecuada representación de cada factor (Lloret-Segura, Ferreres-Traver, HernándezBaeza y Tomás-Marco, 2014).

Tabla 4. Comunalidades de los reactivos por factor del componente Frecuencia de Uso y Función de TIC.

\begin{tabular}{|c|c|c|c|c|}
\hline \multirow[b]{2}{*}{ Reactivos } & \multicolumn{4}{|c|}{ Factores } \\
\hline & $\begin{array}{l}\text { 1. No académico- } \\
\text { compañeros }\end{array}$ & $\begin{array}{l}\text { 2. Académico- } \\
\text { compañeros }\end{array}$ & $\begin{array}{l}\text { 3. No académico- } \\
\text { individual }\end{array}$ & $\begin{array}{l}\text { 4. Académico- } \\
\text { individual }\end{array}$ \\
\hline 21 & .782 & & & \\
\hline 23 & .751 & & & \\
\hline 29 & .625 & & & \\
\hline 18 & .509 & & & \\
\hline 32 & .500 & & & \\
\hline 13 & .494 & & & \\
\hline 19 & .463 & & & \\
\hline 30 & .448 & & & \\
\hline 22 & .407 & & & \\
\hline 1 & & .605 & & \\
\hline 15 & & .596 & & \\
\hline 24 & & .531 & & \\
\hline 25 & & .526 & & \\
\hline 20 & & .453 & & \\
\hline 28 & & .429 & & \\
\hline 7 & & .412 & & \\
\hline 4 & & & .602 & \\
\hline 2 & & & .560 & \\
\hline 8 & & & .530 & \\
\hline 5 & & & .449 & \\
\hline 6 & & & .357 & \\
\hline 27 & & & .351 & \\
\hline 12 & & & & .647 \\
\hline 31 & & & & .647 \\
\hline 17 & & & & .592 \\
\hline 11 & & & & .436 \\
\hline
\end{tabular}


Además del análisis factorial y para profundizar en la relación entre factores, se utilizó el coeficiente de correlación Spearman; para la interpretación de las correlaciones, se tomó en cuenta la tabla de clasificación de Oviedo y Arias (2005).

En cuanto a la relación entre factores, las siguientes correlaciones son significativas al 95\%: A) correlación media y positiva entre los factores No académicocompañeros y Académico-compañeros $\left(r=.447^{* *}\right)$, para los datos de la muestra, lo anterior significa que entre más se usan las TIC para actividades grupales en contextos fuera de la escuela, también se usan más para actividades en colectivo en la Universidad; B) también se obtuvieron correlaciones bajas y positivas entre 1) Académico-individual y No académico-compañeros $\left(r=.197^{* *}\right)$; y 2) Académicocompañeros y académico individual $\left(r=.381^{* *}\right)$, lo anterior indica que a mayor uso de las TIC en la escuela para actividades individuales, aumenta el uso de las tecnologías para actividades en colectivo dentro y fuera de la escuela; C) finalmente se identificaron correlaciones bajas y negativas entre: 1) Académico-compañeros con No académico individual $\left(r=-.310^{* *}\right)$ y 2) No académico individual y Académico individual ( $r=$ $\left.-.132^{* *}\right)$, indicando que a mayor uso de las TIC para actividades individuales fuera del contexto escolar, menos se usarán en actividades individuales o en colectivo relacionadas con la escuela.

El componente Frecuencia de Uso y función de las TIC, obtuvo un Alpha de Cronbach total de .91, lo que equivale a una confiabilidad alta y las confiabilidades por factor (Tabla 5), apuntaron a consistencias medias altas (Celina y Campo, 2005).

Tabla 5. Confiabilidad de los factores del Componente Frecuencia de Uso y Función de las TIC

\begin{tabular}{lc}
\hline Factor & Alpha de Cronbach \\
\hline No académico-compañeros & .844 \\
No académico-individual & .734 \\
Académico-compañeros & .815 \\
Académico-individual & .743 \\
\hline
\end{tabular}

El puntaje mínimo total que puede obtenerse en este componente es 26 y el máximo, 104; a partir del análisis por percentiles, se obtuvieron cuatro categorías de interpretación (Tabla 6).

Tabla 6. Interpretación por percentiles de la puntuación total de Frecuencia de Uso y Función de las TIC

\begin{tabular}{ccc}
\hline Percentiles & $\begin{array}{c}\text { Rangos } \\
\text { (puntajes) }\end{array}$ & $\begin{array}{c}\text { Categorías de } \\
\text { interpretación }\end{array}$ \\
\hline 25 & $26-57$ & 0 a 1 vez por semana \\
50 & $58-62$ & 2 a 3 veces por semana \\
75 & $63-68$ & 4 a 5 veces por semana \\
95 & $69-104$ & Más de 5 veces por semana \\
\hline
\end{tabular}

Finalmente para cada uno de los factores, también se realizó un análisis de percentiles para determinar los puntajes y sus categorías de interpretación (Tabla 7). 
Tabla 7. Percentiles de los factores del componente Frecuencia de Uso y Función.

\begin{tabular}{lcccc}
\hline \multicolumn{1}{c}{ Factores } & \multicolumn{4}{c}{ Percentil } \\
\cline { 2 - 5 } & \multicolumn{1}{c}{50} & \multicolumn{1}{c}{75} & 95 \\
\hline $\begin{array}{l}\text { 1. No académico - } \\
\text { compañeros }\end{array}$ & $9-19$ & $20-24$ & $25-27$ & $28-36$ \\
$\begin{array}{l}\text { 2. No académico - } \\
\text { individual }\end{array}$ & $7-15$ & $16-21$ & $22-25$ & $26-28$ \\
$\begin{array}{l}\text { 3. Académico - } \\
\text { compañeros }\end{array}$ & $6-12$ & $13-14$ & $15-16$ & $17-24$ \\
\begin{tabular}{l} 
4. Académico - individual \\
\hline
\end{tabular} & $4-8$ & $9-10$ & $11-12$ & $13-16$ \\
\hline
\end{tabular}

Nota. Percentil $25=0$ a 1 vez por semana; percentil $50=2$ a 3 veces por semana; el percentil $75=4$ a 5 veces por semana; $y$ el percentil 95 = más de 5 veces por semana.

El componente Contextos Educativos de Uso de las TIC incluye ocho reactivos (Fase 1) y se analiza cualitativamente. Cada respuesta equivale a un punto por lo que se realiza la sumatoria total para cada contexto educativo; él o los contextos con las puntuaciones más altas, se consideran como los de mayor preferencia cotidiana de uso. El contenido de cada reactivo puede complementar el análisis de este componente.

\section{Conclusiones}

En el presente trabajo, se realizó la validación de la Escala Uso y Función de las TIC en Contextos Educativos, la cual quedó conformada por: apartado de datos sociodemográficos; instrucciones; y 34 reactivos que evalúan dos componentes, uno de forma cuantitativa y el otro cualitativa. El primer componente, Frecuencia de Uso y Función de las TIC, incluye 4 factores principales conformados en su mayoría por reactivos positivos, la clasificación de reactivos y la asignación de los mismos a los factores fue a partir del análisis de comunalidades. En relación con lo anterior, se está de acuerdo con Tomás, Sancho, Oliver, Galiana y Meléndez (2012) en que el uso de reactivos positivos e invertidos, disminuye la variabilidad de las puntuaciones debido al error de medición, la deseabilidad social y la aquiescencia. Los cuatro factores de este componente, explican el $42.7 \%$ de la varianza total del instrumento, de acuerdo con Lloret-Segura, Ferreres-Traver, Hernández-Baeza y Tomás-Marcovalor (2014) el resultado es menor al esperado (50 a 60\%) para una escala tipo Likert. Hay que mencionar que el error de medida obtenido para este instrumento fue menor a uno; su cálculo, permitirá a quienes utilicen esta escala, determinar los límites superior e inferior de la puntuación para cada uno de los usuarios.

Los resultados para el componente cuantitativo de confiabilidad total y por factor, se ubicaron en las categorías alta y media alta respectivamente (Celina y Campo, 2005). Los análisis por percentiles realizados para este componente, permitirán la comparación del puntaje natural (total y/o por factor) de una persona, con el grupo normativo de validación; ello permitirá identificar tanto el percentil como la categoría de interpretación que le corresponde a la persona evaluada, lo que facilitará una evaluación más completa para diseñar acciones que favorezcan las áreas de oportunidad en cada usuario. 
Los datos obtenidos por medio de los cuatro factores del componente cuantitativo del instrumento, son complementados con la identificación cualitativa sobre los Contextos Educativos de Uso de las TIC; ambos componentes en conjunto, aportan información para identificar la competencia digital de los universitarios. Ello coincide con lo señalado por Pardo y Cobo (2020) quienes afirmaron que a través de encuestas y estudios cualitativos, es posible identificar la diversidad funcional digital del universo de estudiantes y a partir de ello, realizar la planificación académica y el diseño de estrategias holísticas sobre el uso de las TIC.

Se apoya lo señalado por Prendes et al. (2018) en cuanto a que es necesario llevar a cabo un análisis del contexto y de los aspectos sociales vinculados con las tecnologías aplicadas a la educación así como lo mencionado por Martínez, Steffens, Ojeda y Hernández (2018) sobre la importancia de vincular los componentes sociales y culturales con los tecnológicos para potenciar la educación superior. En la bibliografía existente, que muestra evidencia de que los universitarios distan de utilizar las tecnologías en contextos formales de educación, tal afirmación no puede ser generalizada y dirigir per se la intervención, se precisa evaluar cuál es el uso y función que les otorgan en contextos y momentos específicos, aspecto importante en la identificación de la competencia digital. La identificación de que el uso y función de las TIC se prioriza en contextos informales, podría ser el punto de partida para realizar acciones que favorezcan al ámbito de la educación formal; un ejemplo de ello, fue lo realizado con éxito por Medeiros, Bandeira y Campos (2020) quienes diseñaron un material digital para el aprendizaje de la fibromialgia que fue utilizado por 30 universitarios brasileños a través de sus redes sociales.

En momentos contextuales donde se subraya la educación a distancia, la educación híbrida y la comunicación asíncrona, la evaluación de los universitarios, utilizando instrumentos como el presente, puede favorecer el desarrollo de estrategias de intervención y psicoeducación. Como señalaron Pardo y Cobo (2020) ante la actual emergencia global por Covid-19, los límites de los contextos y los ámbitos de actuación (e.g. personal - escolar) se están desdibujando, lo que podría aprovecharse para naturalizar la cultura de las TIC en la Educación Superior, reto para todos los actores implicados, entre ellos los estudiantes mismos.

A partir de lo anterior, se subraya la importancia de contar con instrumentos confiables (Islas et al., 2016; Madrid et al., 2016; Recio et al., 2020; UNESCO, 2014) que aporten datos para el proceso de evaluación de las TIC en la acreditación de las universidades (Comités Interinstitucionales para la Evaluación de la Educación Superior, 2021; García, Toscano y Álvarez, 2016). En general, la escala que se presenta en este trabajo constituye una herramienta válida y confiable que puede emplearse en las evaluaciones realizadas por las universidades y sus actores sobre el uso de las TIC.

Finalmente, entre las limitaciones de esta investigación, que podrían ser superadas en futuros estudios, está el uso de un muestreo no probabilístico, así como igualar el tamaño de las muestras participantes por cada institución. Para futuras investigaciones, se recomienda continuar con el proceso de estandarización de la presente escala y ampliar su espectro de aplicación. 


\section{Referencias}

Asociación Nacional de Universidades e Instituciones de Educación Superior. (2020). Sugerencias para mantener los servicios educativos curriculares durante la etapa de emergencia sanitaria provocada por el COVID-19. Autor. http://www.anuies.mx/media/docs/avisos/ pdf/200417111353Sugerencias+para+ma ntener+los+servicios+educativos.pdf

Cabero, J. y Llorente, M. C. (2015). Tecnologías de la Información y la Comunicación (TIC): escenarios formativos y teorías del aprendizaje. Revista LaSallista de Investigación, 12(2), 186-193. https://doi.org/10.22507/rli.v12n2a19

Celina, H. y Campo, A. (2005). Aproximación al uso del coeficiente alfa de Cronbach. Revista Colombiana de Psiquiatría, 34(4), 572-580.

https://www.redalyc.org/pdf/806/8063440 9.pdf

Comisión Nacional para la Mejora Continua de la Educación. (2020). 10 Sugerencias para la educación durante la emergencia por COVID-19. Autor. https://www.gob.mx/cms/uploads/attach ment/file/546270/mejoredu_covid-19.pdf

Comités Interinstitucionales para la Evaluación de la Educación Superior. (2021). Acreditación institucional. Autor. https://www.ciees.edu.mx/acreditacion/

Consejo Nacional de Población. (2015). Índice de marginación por entidad federativa $y$ municipio 2015. Anexo B. Autor. https://tinyurl.com/yyu $5 \mathrm{mdr2}$

Coordinación de Tecnologías para la Educación. (2020). Resultados de la octava aplicación del cuestionario diagnóstico sobre habilidades digitales a estudiantes de primer ingreso al Bachillerato de la UNAM. Generación 2020. UNAM. https://educatic.unam.mx/publicaciones/ti cometro/Ticometro-Bachillerato-2019.pdf

Dávila, O. y Gutiérrez, C. (2019). Google Sites como herramienta didáctica online en el aprendizaje significativo del área de ciencia, tecnología y ambiente en estudiantes de cuarto grado de educación secundaria. Hamut'ay, 6(1), 33-53. http://dx.doi.org/10.21503/hamu.v6i1.157 3

Dele-Ajayi, O., Dunsin, O. y Okoli, A. (2021) Teachers' concerns about integrating information and communication technologies in the classrooms. PLoS One, 16(5),

1-19.

https://doi.org/10.1371/journal.pone.0249 703

European Commission. (2019). DigCompOrg. Digitally Competent Educational Organisations. Author. https://ec.europa.eu/jrc/en/digcomporg

European Union. (2018). Recommendations. Key competences for lifelong learning. European reference framework. Official Journal of the European Union, C 189, 1$13 . \quad \mathrm{https}: / /$ eur-lex.europa.eu/legalcontent/EN/TXT/PDF/? uri=CELEX:32018H0604(01)\&amp;rid=7

García, A., Toscano, B. y Álvarez, F. (2016). Acreditación y certificación como avales de calidad en México en programas educativos en TIC. En L. Sánchez, A. García y F. Álvarez (Eds.), El profesional de TIC y la transdisciplinariedad (pp. 60-77). ALFAOMEGA.

https://www.researchgate.net/publication /315785976_Acreditacion_y_Certificacion _como_Avales_de_calidad_en_Mexico_en _Programas_Educativos_en_TIC

Gordon, J., Halász, G., Krawczyk, M., Leney, T., Michel, A., Pepper, D., Putkiewicz, E. y Wisniewski, J. (2010). Key competences in Europe: Opening doors for lifelong learners across the school curriculum and teacher education. CASE. https://dx.doi.org/10.2139/ssrn.1517804

Hernández, V. (2020). Segundo informe de actividades. Gestión 2018 - 2022. UNAM. https://www.zaragoza.unam.mx/wpcontent/Portal2015/InformesGestion/ archivos_gestion/ 2o_informe_Actividades2018-2022.pdf

Instituto Nacional de Estadística y Geografía. (2020). Estadísticas a propósito del día mundial del internet. Datos nacionales. 
Autor.

https://www.inegi.org.mx/contenidos/sala deprensa/aproposito/2020/EAP_Internet2 $0 . p d f$

Islas, C., Franco, S., Delgadillo, O. y Carranza, M. (2016). Descubrimiento de patrones en uso de las TIC por estudiantes universitarios. En J. Prieto y S. Pech. (Eds.), La tecnología como instrumento para potenciar el aprendizaje (pp. 56-63). CIATA. https://doi.org/10.13140/RG.2.1.3802.3920

Jiménez, V. y Alvarado, J. (2017). Validación de un cuestionario diseñado para medir frecuencia y amplitud de uso de las TIC. EDUTEC. Revista Electrónica de Tecnología Educativa, 1(61), 1-14. https://doi.org/10.21556/edutec.2017.61.9 49

Lloret-Segura, S., Ferreres-Traver, A., Hernández-Baeza, A. y Tomás-Marco, I. (2014). El análisis factorial exploratorio de los ítems: una guía práctica, revisada y actualizada. Anales de Psicología, 30(3), 1151-1169.

https://dx.doi.org/10.6018/analesps.30.3.1 99361

Madrid, G., Angulo, J., García, L. y Olivares, C. (2016). Estado del conocimiento en la educación mediada por TIC en México. En J. Prieto y S. Pech. (Eds.), La tecnología como instrumento para potenciar el aprendizaje (pp. 24-31). CIATA. https://doi.org/10.13140/RG.2.1.3802.3920

Martínez, O., Steffens, E., Ojeda, D. y Hernández, H. (2018). Estrategias pedagógicas aplicadas a la educación con medios virtuales para la generación de conocimiento global. Formación Universitaria, 117(5), 11-18. https://dx.doi.org/10.4067/S07185006201800050001

Medeiros, C., Bandeira, C. y Campos, P. (2020). Aula invertida con tecnologías digitales y herramienta metacognitiva para mejorar las clases de educación superior. Revista Latinoamericana de Tecnología Educativa, 19(2),

65-81. https://doi.org/10.17398/1695288X.19.2.65

Mengual, A., Juárez, D., Peydró, M. y Vercher, M. (2013). Las TIC en la formación on line. 3C TIC, 2(5), 1-14. https://doi.org/10.17993/3ctic.2013.25.\%2 0

Olaya, D. y Peirano, F. (2007). El camino recorrido por América Latina en el desarrollo de indicadores para la medición de la sociedad de la información y la innovación tecnológica. Revista Iberoamericana de Ciencia, Tecnología y Sociedad, 3(9), 153-185. https://www.redalyc.org/articulo.oa? id $=92430910$

Olivares, K., Angulo, A., Torres, G. y Madrid, G. (2016). Validación de un modelo de medida para la competencia digital en estudiantes universitarios. En J. Prieto y S. Pech. (Eds.), La tecnología como instrumento para potenciar el aprendizaje (pp. 72-78). CIATA. https://doi.org/10.13140/RG.2.1.3802.3920

Oviedo, H. y Campos-Arias, A. (2005). Aproximación al uso del coeficiente alfa de Cronbach. Revista Colombiana de Psiquiatría, 34(4). 572-580. https://www.redalyc.org/pdf/806/8063440 9.pdf

Organización de las Naciones Unidas para la Educación, la Ciencia y la Cultura. (2014). Metas educativas 2021: desafíos y oportunidades. Autor. https://unesdoc.unesco.org/ark:/48223/pf 0000189945/PDF/189945spa.pdf.multi

Organización de las Naciones Unidas para la Educación, la Ciencia y la Cultura. (2019). Las TIC en la educación. Autor. https://es.unesco.org/themes/ticeducacion

Organización de las Naciones Unidas para la Educación, la Ciencia y la Cultura. (2020). 1.37 billion students now home as COVID19 school closures expand. Author. https://en.unesco.org/news/137-billionstudents-now-home-covid-19-schoolclosures-expand-ministers-scalemultimedia

Pardo, H. y Cobo, C. (2020). Expandir la universidad más allá de la enseñanza remota de emergencia. Outliers School. https://outliersschool.net/wp-content/upl oads/2020/05/Expandir_la_universidad

Prendes, M., Solano, I., Serrano, J., González, V. y Román M. (2018). Entornos personales de aprendizaje para la comprensión y 
desarrollo de la competencia digital: análisis de los estudiantes universitarios en España. Educatio Siglo XXI, 36(2), 115134. http://dx.doi.org/10.6018/j/333081

Ramos, R., Cabrera, G., Calle, F. y Romo, A. (2019). Las TIC en los procesos de enseñanza en la educación superior. Revista Científica Mundo de la Investigación al Conocimiento, 3(3), 706724.

https://doi.org/10.26820/recimundo/3. (3.Esp).noviembre.2019.706-724

Recio, F., Silva, J. y Abricot, N. (2020). Análisis de la competencia digital en la formación inicial de estudiantes universitarios: un estudio de meta-análisis en la Web of Science. Pixel-Bit. Revista de Medios y Educación, (59), 125-146. https://doi.org/10.12795/pixelbit.77759

Secretaria de Desarrollo Social. (2013). Catálogo de localidades. Sistema de apoyo para la planeación del PDZP. Autor. http://www.microrregiones.gob.mx/catloc
/LocdeMun.aspx? tipo $=$ clave $\&$ campo $=$ loc\&ent $=15 \&$ mun $=02$ 5

Secretaría de Economía, Datawheel. (2020). DataMÉXICO BETA. Autor. https://datamexico.org/es/profile/instituti on/universidad

Sistema de Información del Desarrollo Social. (2018). Sistema de información de desarrollo social: delegación Iztapalapa. Autor. https://doi.org/10.4995/thesis/10251/8615 8

Tomás, J., Sancho, P., Oliver, A., Galiana, L. y Meléndez, J. (2012). Efectos de métodos asociados a ítems invertidos vs. ítems en negativo. Revista Mexicana de Psicología, 29(2),

105-115. https://www.redalyc.org/pdf/2430/243030 190001.pdf

Trilla, J., Gros, B., López, F. y Martín, J. (2003). La educación fuera de la escuela. Ámbitos no formales y educación social. Ariel. 
